\title{
UTILIZAÇÃO DOS RECURSOS DO IOS PARA MONITORAR PESSOAS DE TERCEIRA IDADE NA PRÁTICA DE ATIVIDADES FÍSICAS
}

Márcio Rodrigues Lima ${ }^{1}$, Francisco Assis da Silva ${ }^{2}$, Ana Paula Domeneghetti Parizoto Fabrin ${ }^{2}$, Mário Augosto Pazoti ${ }^{2}$, Jair Rodrigues Garcia Júnior ${ }^{3}$

${ }^{1}$ Discente da Faculdade de Informática da UNOESTE; ${ }^{2}$ Docente da Faculdade de Informática da UNOESTE; ${ }^{3}$ Docente da Faculdade de Educação Física da UNOESTE

\section{RESUMO}

O crescimento da população idosa mundial nos dias de hoje representa um importante indicativo da longevidade. O processo de envelhecimento está atrelado às perdas importantes em inúmeras capacidades físicas, as quais levam a diminuição da capacidade funcional e da independência dos idosos. Em trabalhos encontrados na literatura, é possível observar que essas alterações, põem em risco a qualidade de vida dessas pessoas, por limitar a sua capacidade para realizar, com vigor, as suas atividades do cotidiano e colocar em maior vulnerabilidade a sua saúde. A prática de exercícios físicos contribui de maneira significativa para a manutenção da aptidão física do idoso, influenciando, tanto em sua saúde como em suas capacidades funcionais. Entretanto, os exercícios físicos podem apresentar algumas limitações, devido às modificações fisiológicas impostas com o processo de envelhecimento. Foi desenvolvido, neste trabalho, um aplicativo para proporcionar um recurso às pessoas de terceira idade, para que elas possam se exercitar de forma segura com algum controle sobre o que está acontecendo durante essa prática. Esse recurso também se mostra um grande atrativo para essas pessoas, fazendo um bom uso da tecnologia voltada à saúde, promovendo além da segurança, fácil monitoramento fisiológico durante a prática de atividades físicas.

Palavras-chave: iOS; terceira idade; atividade física; smartphone; objective-C; iPhone

\section{USING IOS FEATURES TO MONITOR SENIORS IN PHYSICAL ACTIVITY}

\begin{abstract}
The growth of the elderly population in recent days is an important indicator of longevity. The aging process is linked to important losses in several physical capabilities, which lead to decreased functional capacity and independence of the elderly In studies in the literature, it is observed that these changes put at risk the quality of life of these people, by limiting their ability to perform, with vigor, their daily activities and put in greater vulnerability to your health. The physical exercise contributes significantly to the maintenance of physical fitness of the elderly, influencing both in their health and in their functional capabilities. However, the physical exercises may present some limitations, due to physiological changes imposed on the aging process. An application was developed in this work to provide a resource for people of old age, so that they can exercise safely with some control over what is happening during this practice. This feature also shows a great attraction for these people, making good use of technology focused on health, in addition to promoting safety, easy physiological monitoring during physical activity.
\end{abstract}

Keywords: iOS; seniors; physical activity; smartphone; objective-C; iPhone. 


\section{INTRODUÇÃO}

O crescimento da população idosa mundial nos dias de hoje representa um importante indicativo da longevidade das pessoas, apesar do conhecimento que 0 processo de envelhecimento está atrelado às perdas importantes em inúmeras capacidades físicas, as quais culminam, inevitavelmente, no declínio da capacidade funcional e da independência do idoso (DIAS; GURJÃO; MARUCCI, 2006). Segundo Alves et al. (2004) essas alterações, nos domínios biopsicossociais ${ }^{1}$, põem em risco a qualidade de vida do idoso, por limitar a sua capacidade para realizar, com vigor, as suas atividades do cotidiano e colocar em maior vulnerabilidade a sua saúde.

A prática de exercícios físicos contribui de maneira significativa para a manutenção da aptidão física do idoso, seja na sua vertente da saúde como nas capacidades funcionais. Entretanto, os exercícios físicos podem apresentar algumas limitações para os idosos, devido às modificações fisiológicas impostas com o processo de envelhecimento. O nível de atividade física que é considerada vigorosa para um idoso não pode ser o mesmo que para um adulto saudável (FEDERIGHI, 1995).

Goyaz (2003) mostra em seu trabalho

\footnotetext{
${ }^{1}$ Biopsicossociais: é a visão integral do ser e do adoecer que compreende as dimensões física, psicológica e social (MARCO, 2006).
}

a importância das atividades físicas e de lazer para a qualidade de vida na terceira idade, e algumas possibilidades de se buscar o equilíbrio entre as potencialidades e as limitações decorrentes das transformações impostas pelo processo de envelhecimento (como queda da força muscular, diminuição do débito cardíaco, diminuição da densidade óssea e da massa muscular, diminuição de tamanho e número de neurônios, diminuição na velocidade de condução nervosa, dentre outras), por meio de uma vida mais ativa. $\mathrm{O}$ autor também afirma que as atividades direcionadas ao idoso devem ser organizadas considerando as suas particularidades e realizadas de forma gradual. Um estilo de vida ativo traz efeitos benéficos para a manutenção da capacidade funcional e da autonomia física durante o processo de envelhecimento. No entanto, antes de iniciar qualquer tipo de exercício físico é importante realizar uma avaliação com um profissional de educação física, a fim de definir as atividades físicas mais condizentes com suas possibilidades e limitações.

Neste trabalho, foi desenvolvido um recurso tecnológico, tendo em vista uma grande preocupação com a saúde das pessoas idosas, para auxílio na prática de atividades físicas de forma individual. Desejase com isso auxiliar no controle da intensidade dos exercícios praticados, dentro 
dos limites impostos pelo organismo de cada pessoa e, caso uma pessoa venha a passar mal, algum conhecido será informado sobre sua localização, visando facilitar o seu socorro.

As demais seções deste trabalho estão organizadas da seguinte maneira: na Seção 2 são apresentados os recursos necessários para realizar o monitoramento fisiológico; na Seção 3 é apresentada a forma como a aplicação foi desenvolvida e a metodologia utilizada na definição de suas funcionalidades; na Seção 4 são apresentados os experimentos realizados para verificar a eficácia da aplicação desenvolvida; na Seção 5 são apresentados os resultados obtidos com os experimentos realizados e a eficácia da aplicação desenvolvida; por fim, a Seção 6 apresenta as conclusões e algumas sugestões para trabalhos futuros.

\section{PARÂMETROS}

FISIOLÓGICOS

\section{MONITORADOS}

Alguns autores consideram como atividade física qualquer movimento corporal produzido pela musculatura esquelética que resulta em gasto energético e o sedentarismo no lazer, como a não realização de atividades físicas. A prática de atividades físicas de forma regular reduz o risco de doença cardiovascular, alguns tipos de cânceres, auxilia no controle da pressão arterial e no diabetes tipo II, promovendo o aumento do turnover (rotatividade) da insulina, por maior captação hepática e, melhora a sensibilidade dos receptores periféricos, reduzindo as complicações relacionadas com as comorbidades $^{2}$. Além disso, a prática de atividade física, associada à dieta, melhora o perfil lipídico a longo prazo (CUNHA et al., 2008).

Uma das mais evidentes alterações que acontecem com o aumento da idade cronológica é a mudança nas dimensões corporais. Com o processo de envelhecimento existem mudanças principalmente na estatura, no peso e na composição corporal. Apesar do alto componente genético no peso e na estatura dos indivíduos, outros fatores, como dieta e atividade física, fatores psicossociais e doenças, entre outros, estão envolvidos nas alterações desses dois componentes durante o envelhecimento. Há uma diminuição da estatura com o passar dos anos, por causa da compressão vertebral, do estreitamento dos discos e da cifose (MATSUDO, 2002).

\section{Levando em consideração a} preocupação com o bem estar das pessoas de terceira idade na realização de atividades físicas, este trabalho provê um mecanismo

\footnotetext{
${ }^{2}$ Co-morbilidade, ou diagnóstico duplo, é definida pela Organização Mundial de Saúde (OMS) como a co-ocorrência no mesmo indivíduo de uma disfunção por consumo de substâncias psicoativas e uma outra perturbação psiquiátrica (OMS, 1995).
} 
de monitoramento à essas pessoas, visando evitar que os seus limites fisiológicos sejam excedidos, e assim evitar riscos à sua saúde. Utilizando essa solução, as pessoas podem obter dados fisiológicos sobre seu corpo, como índice de massa corporal (IMC), frequência cardíaca e gasto calórico, com base na atividade física exercida e dados previamente cadastrados (peso, altura e data de nascimento). Ao executar uma atividade física ao ar livre, também é possível informar a distância percorrida, o tempo de duração da atividade executada, e até mesmo o percurso. No trabalho, também foi desenvolvido um recurso que identifica uma interrupção não esperada da atividade física, interpretando-a como um possível acidente, e nesse caso, por meio de uma mensagem SMS (serviço de troca de mensagens via dispositivos móveis, através da rede de comunicação de telefonia móvel), avisa outras pessoas sobre a sua localização, visando facilitar o socorro e consequentemente ajudando a salvar vidas.

Neste trabalho é utilizado uma cinta peitoral para capturar a frequência cardíaca de uma pessoa, em conjunto com uma aplicação definida e implementada para ser executada na plataforma iOS, que é o sistema operacional do iPhone (MILANI, 2012). A cinta peitoral (60beat Chest Strap) captura a frequência cardíaca da pessoa e a envia para um dispositivo (60beat Heart Rate RX) conectado à entrada do fone de ouvido do iPhone. A aplicação se comunica com esse dispositivo obtendo assim os dados da frequência cardíaca. Embora tenha sido utilizado apenas estes equipamentos, também é possível utilizar uma cinta peitoral que transmite os dados via conexão Bluetooth ${ }^{3}$, dispensando a utilização do dispositivo conectado ao iPhone.

A partir da equação de Karvonen (MARQUES, 2009) que utiliza a idade da pessoa, a aplicação calcula (estima) a FC máxima (frequência cardíaca máxima), subtraindo a idade da pessoa em anos do valor padrão 220, possibilitando posteriormente obter a FC min e a FC max de treinamento (zona de treinamento) necessárias para definir limites onde a queima de gordura e o condicionamento cardiovascular sejam eficazes (intensidades superiores a esse limite provocam desconforto e antecipam a fadiga). Utilizando esses cálculos juntamente com os dados da frequência cardíaca obtidos pela cinta peitoral, a aplicação emite avisos à pessoa quando a FC max de treinamento for atingida ou ultrapassada.

A partir dos dados peso e altura, previamente informados pelo usuário, a

\footnotetext{
${ }^{3}$ Bluetooth: é uma tecnologia de rádio para comunicações de curto alcance, com baixo consumo de energia e baixo custo, favorecendo o seu emprego na implementação de redes ad hoc (dispensam o uso de um ponto de acesso comum) (PRIESS, 2003).
} 
aplicação calcula o índice de massa corporal (IMC), que informa o grau de adequação do peso de uma pessoa à sua altura, indicando eutrofia (estado nutricional adequado, em que não há déficit ou excesso de peso), sobrepeso ou obesidade (excesso de peso). Segundo o Ministério da Saúde, o excesso de peso corporal pode ser estimado por diversos métodos ou técnicas, como pregas cutâneas, relação cintura-quadril, ultra-som, ressonância magnética, entre outras. Entretanto, neste trabalho o cálculo do IMC foi utilizado, devido a sua simplicidade de obtenção e correlação com a gordura corporal.

$\mathrm{Na}$ aplicação estão pré-definidas algumas atividades físicas associadas com o valor MET (expressão da intensidade do metabolismo em repouso em um dado momento) correspondente à cada atividade. Esses dados foram definidos conforme informações disponibilizadas pela Compendium of Physical Activities ${ }^{4}$, e juntamente com o peso da pessoa e o tempo de duração da atividade física, permitem que a aplicação calcule o gasto energético e ou calórico. A Tabela 1 apresenta os valores MET referentes às atividades físicas utilizados neste trabalho.

\footnotetext{
${ }^{4}$ Compendium of Physical Activities: realiza estudos e disponibiliza um esquema de codificação que classifica atividades físicas específicas pela taxa de gasto energético. Essas classificações podem ser encontradas em: https://sites.google.com/site/compendiumofphysicalactivities/Activ ity-Categories.
}

Tabela 1. Atividades físicas selecionadas para este trabalho com 0 valor MET correspondente.

\begin{tabular}{l|c}
\hline Descrição & MET \\
$\begin{array}{l}\text { Passear com um cão, ir para o } \\
\text { trabalho }\end{array}$ & 3,0 \\
\hline $\begin{array}{l}\text { Caminhar a uma velocidade de } 4,8 \\
\mathrm{~km} / \mathrm{h}\end{array}$ & 4,0 \\
\hline $\begin{array}{l}\text { Caminhada a uma velocidade de } 6,4 \\
\mathrm{~km} / \mathrm{h}\end{array}$ & 5,0 \\
\hline $\begin{array}{l}\text { Caminhada a uma velocidade de } 7 \\
\mathrm{~km} / \mathrm{h}\end{array}$ & 6,3 \\
\hline $\begin{array}{l}\text { Correr a uma velocidade de } 8 \mathrm{~km} / \mathrm{h} \\
\text { Correr a uma velocidade de } 8 \mathrm{~km} / \mathrm{h} \\
\text { em terreno irregular }\end{array}$ & 8,6 \\
\hline $\begin{array}{l}\text { Correr a uma velocidade de } 9,7 \\
\mathrm{~km} / \mathrm{h}\end{array}$ & 10,0 \\
\hline Correr a uma velocidade de $12 \mathrm{~km} / \mathrm{h}$ & 12,5 \\
\hline
\end{tabular}

\subsection{Frequência Cardíaca}

\subsubsection{Frequência Cardíaca Máxima}

O débito cardíaco máximo tende a diminuir de forma linear tanto nos homens quanto nas mulheres após os trinta anos de idade. Isto se deve, sobretudo, à diminuição da frequência cardíaca máxima com a idade. Por exemplo, como o débito cardíaco é igual à frequência cardíaca multiplicada pelo volume de ejeção, qualquer diminuição da frequência cardíaca acarreta diminuição do débito cardíaco. A diminuição da frequência cardíaca máxima com a idade pode ser calculada usando a Equação (1) (POWERS; HOWLEY, 2000).

$$
F C \_M a x=220-\text { idade }(\text { anos })
$$




\subsubsection{Zona de Treinamento}

A zona de treinamento corresponde ao intervalo entre a frequência cardíaca (FC) de treinamento mínima (50\% da FC máxima, que representa um estímulo suficiente para induzir adaptações sistêmicas e musculares) e a frequência cardíaca de treinamento máxima (70\% da FC máxima, que representa o limite de condicionamento aeróbico do individuo, acima do qual o esforço passa a ser predominantemente anaeróbico e não pode ser mantido por tempo prolongado, mesmo havendo adaptações). As frequências cardíacas de treinamento mínima e máxima podem ser calculadas utilizando a Equação (2).

$$
\begin{aligned}
& F C_{-} \text {trein_min }=F C_{-} \text {Max }^{*} 0,50 \\
& F C_{\text {_trein_max }}=F C_{-} \text {Max } * 0,70
\end{aligned}
$$

Segundo McArdle, Katch e Kacth (2011), a capacidade aeróbia melhora se a atividade física for de intensidade suficiente para fazer aumentar a frequência cardíaca até pelo menos $70 \%$ da frequência cardíaca máxima ( $F C \max$ ).

Com a utilização de um monitor cardíaco, juntamente com a data de nascimento previamente informada, é possível identificar se os batimentos cardíacos de uma pessoa estão dentro da zona de treinamento, e caso ultrapasse o limite máximo, será informado à pessoa sobre esse aumento, evitando risco à saúde.

\subsection{Gasto Energético e ou Calórico}

O equivalente metabólico corresponde ao consumo de $3,5 \mathrm{ml}$ de $\mathrm{O}_{2}$ por $k g$ peso corporal por minuto ( $3,5 \mathrm{ml} / \mathrm{kg} / \mathrm{min}$ ), que representa a média de consumo com o indivíduo estando em repouso. Quando se realiza um esforço, o consumo de $\mathrm{O}_{2}$ aumenta e, a relação da magnitude desse aumento com o consumo durante o repouso é dada pelo valor do MET. Assim, se o valor em MET de um exercício é de 2,5 ( $M E T=2,5)$ significa que o esforço é 2,5 vezes maior que o gasto em repouso.

Existem tabelas com valores em MET para vários exercícios e atividades físicas (AINSWORTH et al., 2000). Algumas atividades podem ser observadas na Tabela 1. Dessa forma, o cálculo do gasto energético de uma atividade $(k c a l)$ pode ser realizado conforme a Equação (5).

Gasto energético atividade $(\mathrm{kcal})=\mathrm{MET} * \operatorname{peso}(\mathrm{kg}) *$ tem

\section{4. Índice de Massa Corporal}

A avaliação da composição corporal é uma medida importante do estado nutricional de indivíduos. Um dos métodos mais simples para essa avaliação é o cálculo 
do índice de massa corporal (IMC), que informa o grau de adequação do peso em relação a altura de uma pessoa. Esse cálculo é obtido pela divisão do peso (em quilogramas) pela altura (em metros), elevado ao quadrado $\left(\mathrm{kg} / \mathrm{m}^{2}\right)$.

Segundo Nunes et al. (2001), a Organização Mundial de Saúde estabelece os valores de limites para classificação divididos da seguinte maneira: (1) magras: IMC abaixo de 18,5; (2) normais: IMC entre 18,5 e 24,9; (3) sobrepeso: IMC entre 25 e 29,9; (4) obesas: IMC igual ou maior do que 30.

\subsection{Distância Percorrida}

A equação de Haversine (6) leva em consideração a circunferência da terra para calcular a distância entre dois pontos a partir da latitude e a longitude destes dois pontos. Utilizada por navegadores antigos, essa forma é facilitada pelos recursos computacionais atuais.

$$
\begin{gathered}
a=\sin ^{2}(\Delta l a t / 2)+\cos (\text { lat } 1) * \cos (\text { lat } 2) * \sin ^{2}(\text { Llong / }) \\
c=2 * a \tan 2(\sqrt{a}, \sqrt{(1-a)}) \\
d=R * c
\end{gathered}
$$

Considera-se $R$ como o maior raio da Terra estabelecido como $6.378,137 \mathrm{~km}$ na linha do equador para o DATUM WGS84. DATUM refere-se ao modelo matemático teórico da representação da superfície da Terra ao nível do mar utilizado pelos cartógrafos numa dada carta ou mapa. O $\Delta l a t$ é a diferença entre as duas latitudes utilizadas e o slong é o mesmo padrão, apenas referente à longitude (ASSIS, 2010).

\section{METODOLOGIA UTILIZADA PARA}

\section{MONITORAMENTO DE ATIVIDADES FÍSICAS}

\subsection{Delimitação da zona de treinamento}

Durante a prática de uma atividade física, ao ser capturada a frequência cardíaca, esta é comparada à zona de treinamento calculada pela aplicação a partir da idade (previamente informada) da pessoa praticante. Nessa comparação, a aplicação emite um alerta, caso o usuário ultrapasse o limite máximo da zona de treinamento (seção 2.1.2), visando evitar riscos à saúde. $A$ Figura 1 ilustra a representação gráfica na aplicação da frequência cardíaca capturada, associada a zona de treinamento.
(a)
9 ( (b)
$1:$ (c)
1 t

Figura 1. (a) frequência cardíaca abaixo da zona de treinamento. (b) frequência cardíaca dentro da zona de treinamento. (c) frequência cardíaca acima da zona de treinamento.

\subsection{Detecção da interrupção de uma atividade física}


A aplicação definida e implementada neste trabalho identifica uma interrupção não esperada da atividade física praticada, que é realizada por meio de dados obtidos a partir do sensor acelerômetro ${ }^{5}$ presente no iPhone. Esse sensor relata valores para cada um dos eixos $(X, Y$ e $Z)$ em unidades de forçag (força gravitacional), sendo o valor 1,0 a representação da aceleração de cerca de $+1 \mathrm{~g}$ (unidade de aceleração) ao longo de um determinado eixo. Cada evento de aceleração inclui leituras simultâneas ao longo dos três eixos do dispositivo (APPLE, 2012). A Figura 2 apresenta a representação dos eixos do acelerômetro.

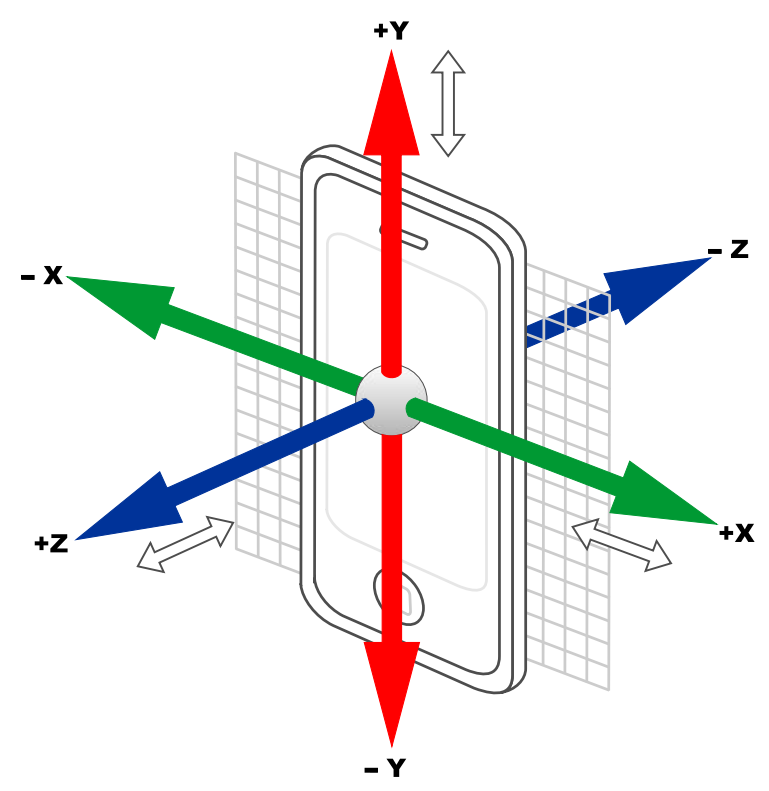

Figura 2. Representação dos eixos do acelerômetro

\footnotetext{
${ }^{5}$ Acelerômetro: é um instrumento capaz de medir a aceleração sobre objetos, calculando qualquer força exercida sobre ele (OLIVEIRA, 2005).
}

A pessoa praticante ao movimentarse durante a execução de uma atividade física, com o equipamento junto ao seu corpo (em um bolso ou preso a um dos braços), o sensor acelerômetro apresenta valores que indicam a força exercida para a movimentação do equipamento. A partir da utilização desses valores torna-se possível identificar se a pessoa está se movimentando, quando o valor apresentado por um dos eixos do acelerômetro for igual ou superior a 1,17. Esse valor foi definido a partir de experimentos realizados, sendo que todos os eixos com valores inferiores a esse, indica que há uma interrupção da atividade física praticada.

Quando a pessoa praticante para de se movimentar, consequentemente o mesmo acontece com o equipamento. Essa parada é interpretada na aplicação desenvolvida como um possível acidente. Para que isso não aconteça é necessário pausar o monitoramento da atividade física manualmente. Quando a interrupção da atividade física é identificada, uma mensagem é exibida na aplicação seguida da emissão de um aviso sonoro. Essas notificações são apresentadas durante 6 segundos, ou até que a pessoa praticante pause o monitoramento da atividade física manualmente. A Figura 3 apresenta a 
mensagem exibida na aplicação quando a interrupção da atividade física é detectada.

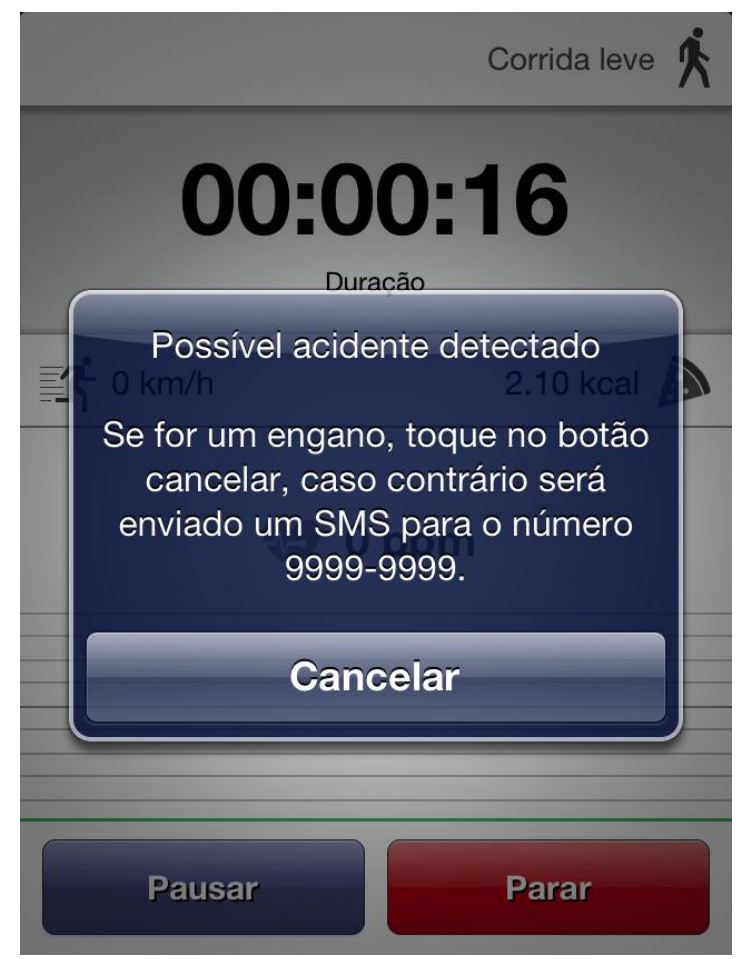

Figura 3. Mensagem de alerta informando a detecção de um possível acidente.

Quando o tempo de espera pela interrupção manual da atividade física é atingindo, por meio de uma mensagem SMS enviada automaticamente, a aplicação avisa algum conhecido (previamente definido) sobre a possibilidade da ocorrência de um acidente com a pessoa praticante.

Antes da mensagem SMS ser enviada, a aplicação acessa os Serviços de Localização do iOS e captura os dados da localização geográfica do praticante (latitude e longitude), e cria com esses uma $\mathrm{URL}^{6}$ que

\footnotetext{
${ }^{6}$ URL (Uniform Resource Locator): é o endereço de um recurso disponível em uma rede de computadores (RFC 1738).
}

permite acesso ao Google Maps (conforme mostra a Figura $4(b))$, exibindo a localização da pessoa em um mapa. Após a criação da URL, esta é anexada a mensagem SMS que é enviada. A Figura 4 (a) mostra uma mensagem SMS enviada pela aplicação informando um acidente. A Figura 4 (b) mostra um mapa com a indicação da localização da pessoa acidentada. 

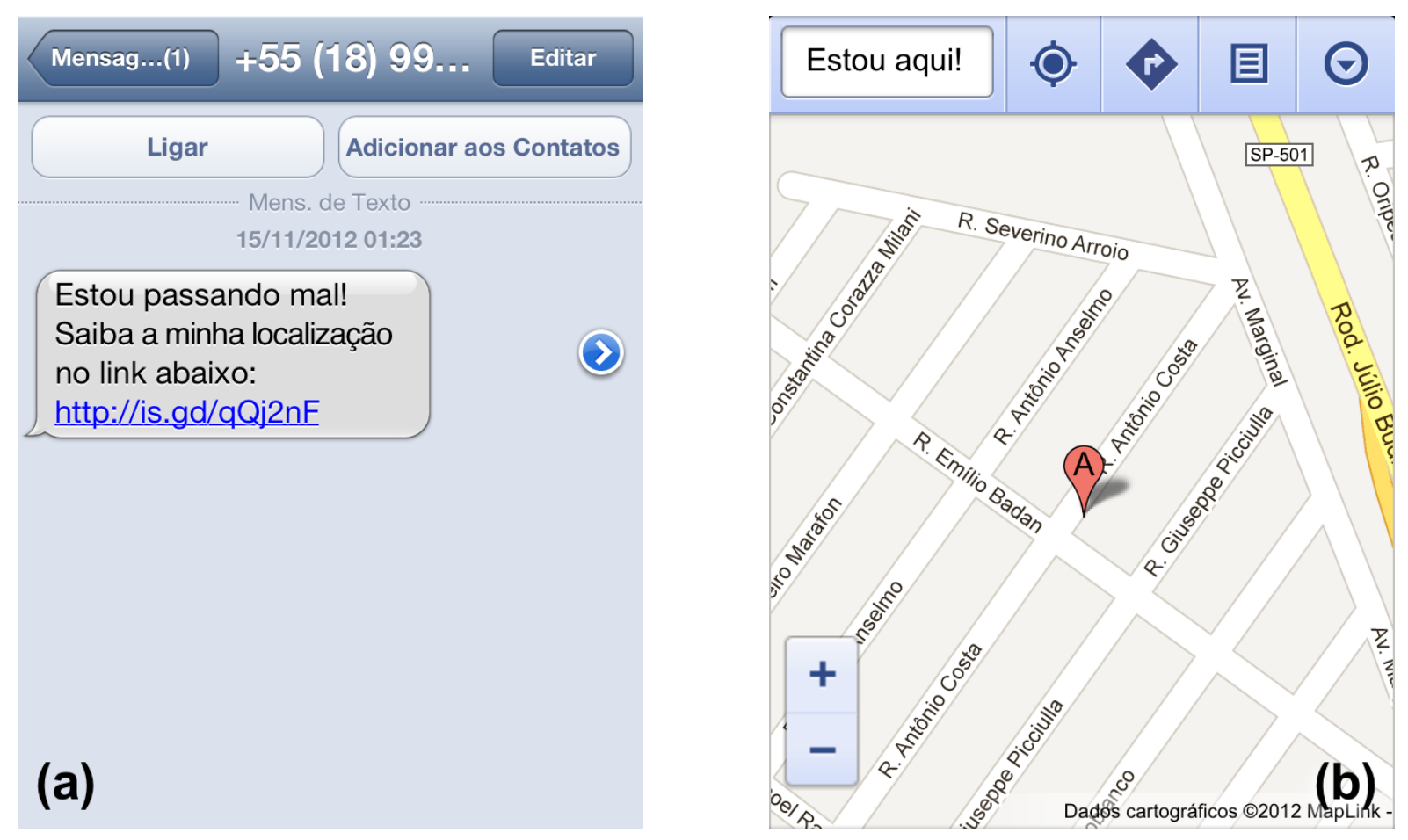

Figura 4. (a) Mensagem SMS enviada informando um acidente juntamente com o link para a localização da pessoa acidentada; (b) Mapa com a localização da pessoa acidentada com acesso pelo link enviado na mensagem SMS.

Devido a limitação de caracteres no envio da mensagens SMS, foi utilizado na aplicação desenvolvida um recurso para reduzir o tamanho da URL gerada para acesso ao Google Maps. Para isso, a aplicação utiliza um serviço gratuito disponibilizado pelo Web Site http://is.gd para gerar uma URL reduzida. A Figura 5 mostra a diferença na quantidade de caracteres entre as URLS geradas.

\begin{tabular}{|cc|}
\hline http://maps.google.com/ & \\
?\|l=-22.142995,- & \\
$51.173066 \& q=$ Estou+ & \\
aqui!@-22.142995,- & http://is.gd/wPre \\
51.173066 & By \\
(a) & (b)
\end{tabular}

Figura 5. (a) URL gerada pela aplicação para exibição da localização da pessoa cidentada no Google Maps. (b) URL reduzida gerada pelo serviço disponível em http://is.gd.

\subsection{Web Services para fornecer dados para outras aplicações}

A aplicação definida e implementada neste trabalho possibilita por meio de um 
Web Service o fornecimento de informações da pessoa (altura, peso, idade, frequência cardíaca máxima e zona de treinamento) e informações da pessoa em relação a atividade física escolhida (frequência cardíaca e gasto calórico). Essas informações podem ser utilizadas por alguma aplicação que as necessite em tempo real para auxiliar no monitoramento durante a prática de alguma atividade física.

O Web Service é executado, quando solicitado, juntamente com a aplicação de monitoramento de atividades físicas desenvolvido neste trabalho. Para a execução desse serviço, faz-se necessário ter uma rede Wi- $\mathrm{Fi}^{7}$ disponível e que o iPhone esteja com uma conexão estabelecida junto a ela. Podese exemplificar o uso desse recurso para atividades aeróbicas em que o praticante esteja repetindo movimentos e deseja acompanhar a sua situação fisiológica durante a prática dessa atividade. A Figura 6 mostra um cenário para prática dessas atividades, contendo um sensor Kinect (usado no vídeo game X-Box da Microsoft capaz de reconhecer a estrutura do corpo humano e seus movimentos) conectado a um computador, com acesso a rede local via conexão com um dispositivo Wi-Fi. Ainda

\footnotetext{
${ }^{7} \mathrm{Wi}-\mathrm{Fi}$ : é o nome dado a uma rede sem fios que utiliza ondas de rádio de baixa frequência para transmitir dados em alta velocidade em áreas limitadas. O termo Wi-Fi é uma abreviação de "Wireless Fidelity", empregado para descrever produtos que seguem o conjunto de padrões 802.11 desenvolvido pelo Institute of Electrical and Electronic Engineers - IEEE (ZEINDIN, 2003).
}

nesta figura, pode ser visualizado um iPhone conectado na mesma rede local que 0 computador. 


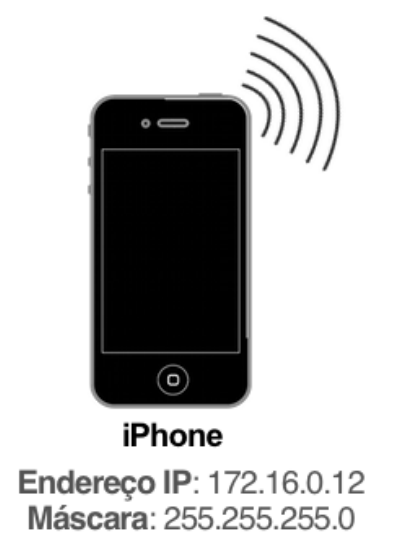

Endereço IP: 172.16 .0 .12

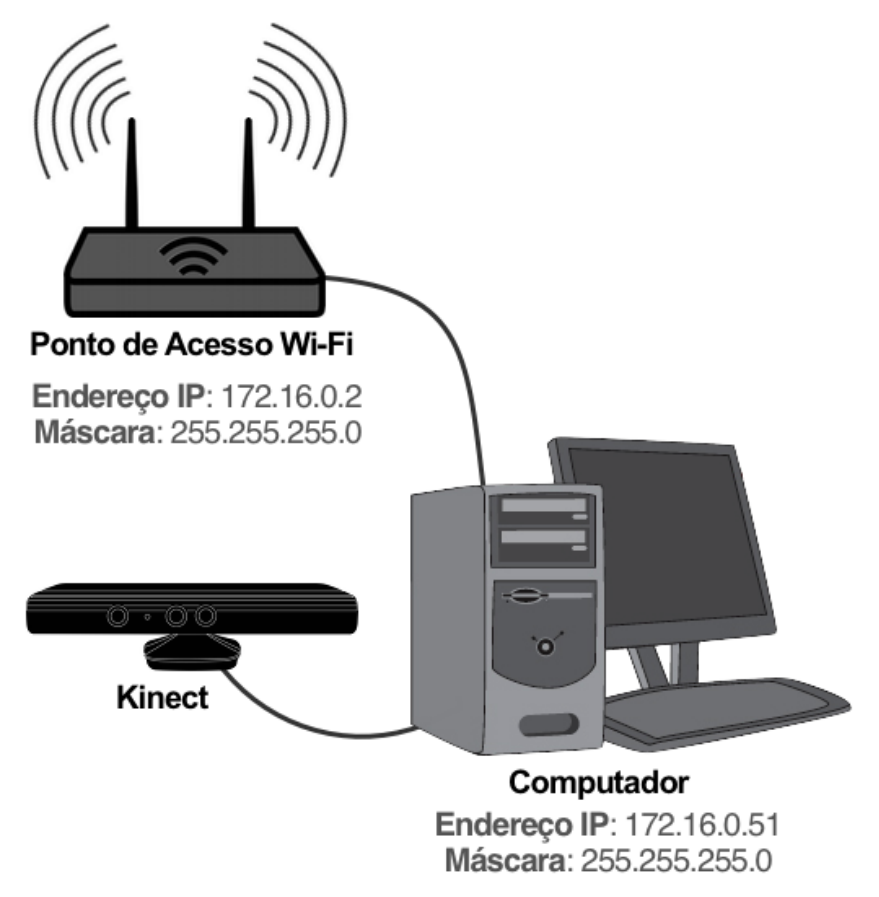

Figura 6. Transmissão de dados para aplicação externa.

No cenário apresentado na Figura 6, uma suposta aplicação desenvolvida para o Kinect busca os dados da pessoa praticante da atividade física e atua no auxilio do controle sobre as limitações fisiológicas (seção 2), para que a atividade seja realizada de forma mais segura e eficiente. Essas preocupações são mais relevantes quando se trata da prática de atividades físicas realizadas por pessoas idosas.

Ao iniciar o Web Service no iPhone, esse pode ser acessado por meio do endereço $\mathbb{I P}^{8}$ atribuído no momento da conexão Wi-Fi, juntamente com a porta 8081, definida na aplicação. As informações

\footnotetext{
${ }^{8}$ Internet Protocol (IP): é a identificação única de um dispositivo em uma rede computadores. É o meio utilizado para a comunicação entre dispositivos interconectados em uma rede (RFC 791).
}

da pessoa e da atividade (frequência cardíaca e gasto calórico) são obtidas por meio de uma solicitação HTTP $^{9}$ padrão, e são recuperadas no formato de arquivo $\mathrm{XML}^{10}$. $\mathrm{A}$ Figura 6 mostra as informações da pessoa referente a data de nascimento, idade, peso, sexo, frequência cardíaca máxima, frequência cardíaca de treinamento máxima e frequência cardíaca treinamento mínima.

\footnotetext{
9 Hypertext Transfer Protocol (HTTP) é um protocolo de comunicação na camada de aplicação, utilizado por sistemas de informação de hipermídia, distribuídos e colaborativos (RFC 2616).

${ }^{10}$ Extended Markup Language (XML) é uma versão abreviada do SGML (Standard Generalized Markup Language), que possibilita ao autor especificar a forma dos dados no documento, além de permitir definições semânticas (ALMEIDA, 2002).
} 


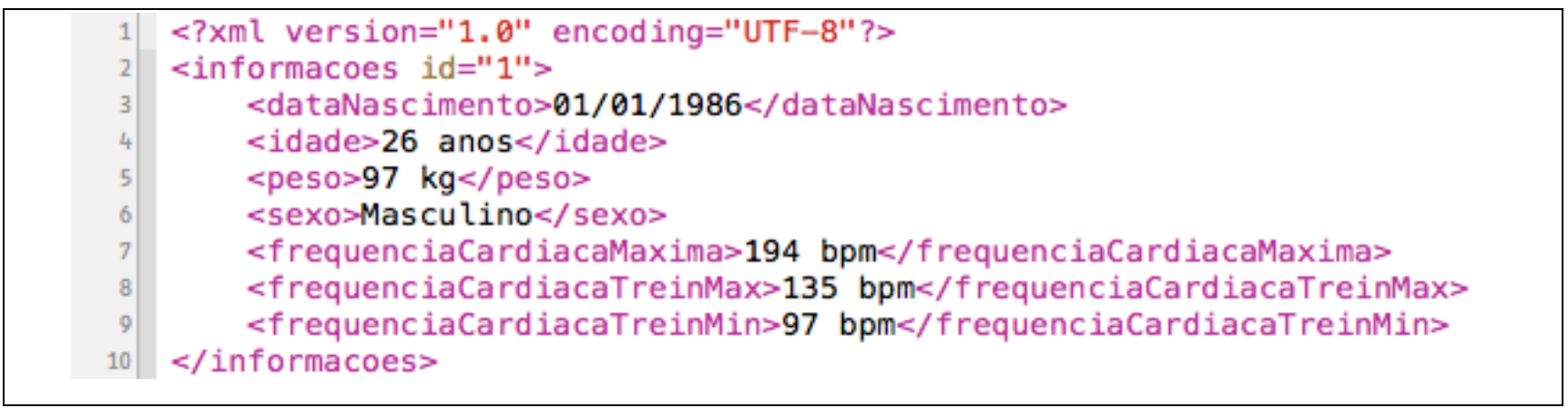

Figura 6. XML com as informações da pessoa praticante.

A Figura 7 mostra as informações da cardíaco, a data e horário que foram pessoa referente ao valor do batimento capturadas.

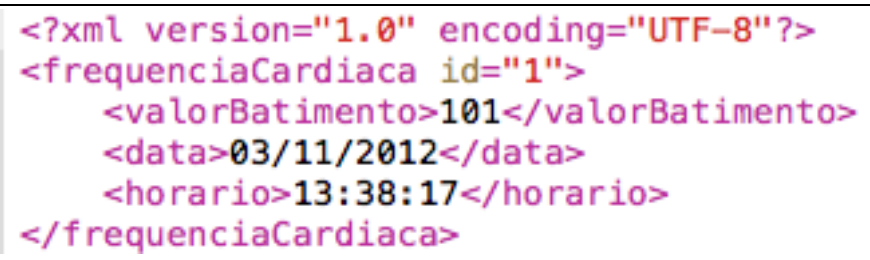

Figura 7. XML com as informações da frequência cardíaca da pessoa praticante capturada durante a prática uma atividade física.

A Figura 8 mostra as informações da pessoa referente ao valor do gasto calórico, a data e horário que foram capturadas.

\section{EXPERIMENTOS}

Neste trabalho, foram realizados experimentos para verificar a eficácia da $<$ ?xml version $=" 1.0$ " encoding $="$ UTF -8 <gastoCalorico $i d=" 1 ">$ $<$ valorKcal $>27.516</$ valorKcal> $<$ data $>03 / 11 / 2012</$ data $>$ $<$ horario $>13: 38: 42</$ horario $>$ $</$ gastoCalorico $>$

Figura 8. XML com as informações do gasto calórico da pessoa praticante calculado durante a prática uma atividade física. aplicação, se os dados estão sendo devidamente capturados pelos sensores e a coerência dos cálculos realizados durante a execução de uma atividade física.

Nos experimentos, uma pessoa com 26 anos de idade, utilizando os equipamentos (sensores) realizou três vezes a mesma atividade física, em três cidades (Martinópolis, Presidente Prudente e São Paulo) ao redor de um quarteirão. A partir da 
segunda execução da atividade física foram acrescentados 20 anos na idade da pessoa. A Figura 9 mostra a representação de como a atividade física foi praticada nas cidades citadas com o ajuste realizado na idade da pessoa praticante.

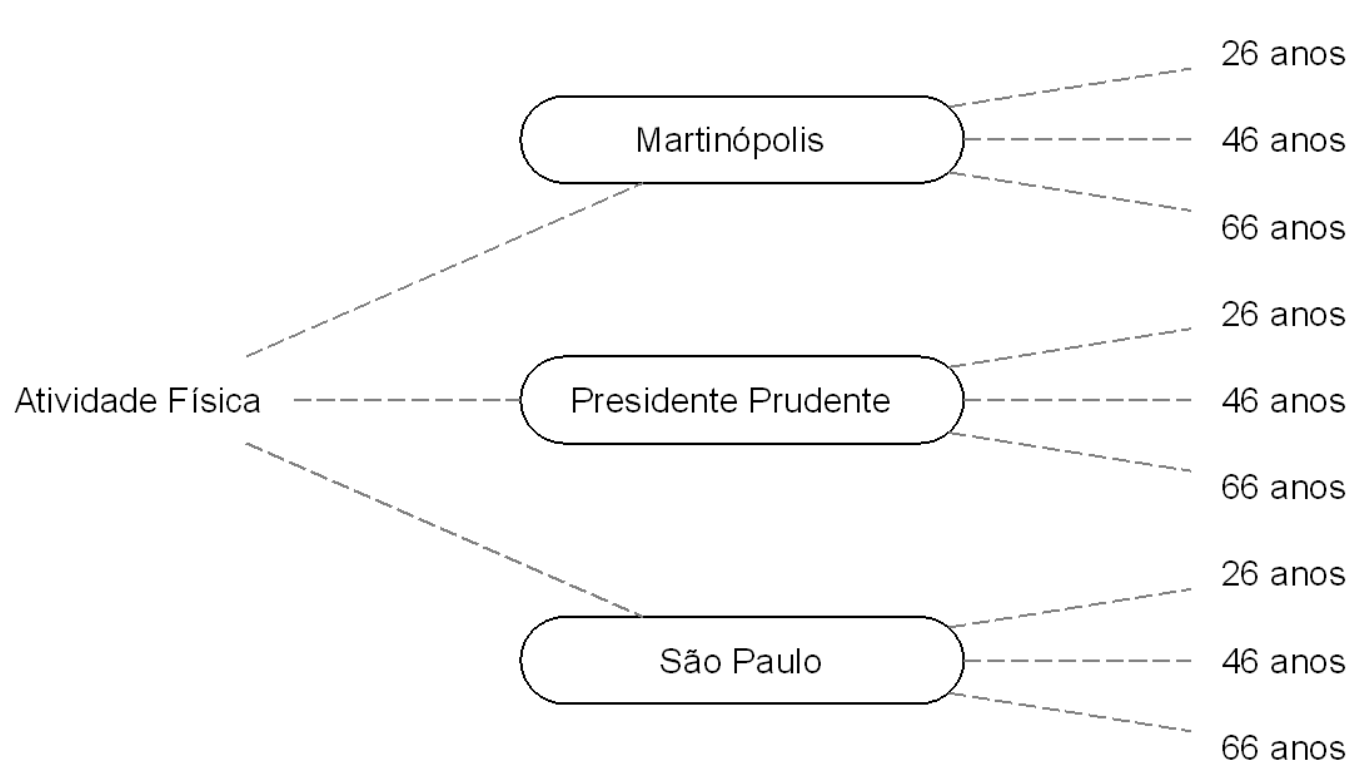

Figura 9. Representação da atividade física praticada em três cidades com ajuste na idade da pessoa praticante.

\section{RESULTADOS}

A partir dos resultados obtidos com os experimentos realizados, foi possível observar que em alguns casos, ao iniciar uma atividade física, os valores obtidos referentes à frequência cardíaca e a velocidade de deslocamento apresentam uma elevação ou queda drástica (representação de valores não condizentes com a realidade daquele determinado momento), e após alguns instantes (aproximadamente cinco segundos) os valores são estabilizados. Na Figura 10 é apresentado o percurso realizado na cidade de Martinópolis em uma atividade física praticada três vezes (percurso A, B e C), nesta, as linhas representando o trajeto apresentam algumas diferenças, provocadas pelo fato da pessoa não percorrer exatamente o mesmo caminho e não manter uma velocidade constante. A Tabela 2 mostra as coordenadas geográficas capturas durante a prática da atividade física mencionada, que foram utilizadas para desenhar o percurso na Figura 10. 
Tabela 2. Informações do percurso capturadas durante a prática de uma atividade física.

\begin{tabular}{c|c|c|c|c|c}
\multicolumn{2}{c|}{ Percurso A } & \multicolumn{2}{c|}{ Percurso B } & \multicolumn{2}{c}{ Percurso C } \\
\hline Latitude & Longitude & Latitude & Longitude & Latitude & Longitude \\
\hline-22.142995 & -51.173066 & -22.142949 & -51.173138 & -22.143069 & -51.173136 \\
\hline-22.142869 & -51.173121 & -22.142839 & -51.173086 & -22.142880 & -51.173104 \\
\hline-22.142751 & -51.172949 & -22.142788 & -51.172904 & -22.142798 & -51.173035 \\
\hline-22.142642 & -51.172758 & -22.142775 & -51.172719 & -22.142760 & -51.172843 \\
\hline-22.142914 & -51.172644 & -22.142904 & -51.172598 & -22.142767 & -51.172686 \\
\hline-22.143095 & -51.172511 & -22.143096 & -51.172535 & -22.142868 & -51.172623 \\
\hline-22.143267 & -51.172406 & -22.143302 & -51.172437 & -22.142989 & -51.172535 \\
\hline-22.143460 & -51.172319 & -22.143481 & -51.172343 & -22.143248 & -51.172457 \\
\hline-22.143659 & -51.172269 & -22.143688 & -51.172267 & -22.143477 & -51.172357 \\
\hline-22.143787 & -51.172433 & -22.143798 & -51.172427 & -22.143630 & -51.172280 \\
\hline-22.143907 & -51.172709 & -22.143869 & -51.172594 & -22.143772 & -51.172333 \\
\hline-22.143992 & -51.172937 & -22.143922 & -51.172786 & -22.143852 & -51.172539 \\
\hline-22.143871 & -51.173105 & -22.143965 & -51.173046 & -22.143927 & -51.172749 \\
\hline-22.143670 & -51.173218 & -22.143805 & -51.173153 & -22.143972 & -51.172952 \\
\hline-22.143428 & -51.173294 & -22.143604 & -51.173216 & -22.143903 & -51.173090 \\
\hline-22.143208 & -51.173399 & -22.143390 & -51.173293 & -22.143707 & -51.173159 \\
\hline-22.143000 & -51.173471 & -22.143206 & -51.173371 & -22.143491 & -51.173252 \\
\hline-22.142919 & -51.173298 & -22.142999 & -51.173458 & -22.143332 & -51.173338 \\
\hline-22.142945 & -51.173137 & -22.142907 & -51.173243 & -22.143127 & -51.173420 \\
\hline-22.143036 & -51.173081 & -22.142986 & -51.173110 & -22.142973 & -51.173432 \\
\hline & & & & -22.142898 & -51.173241 \\
\hline & & & & -22.142962 & -51.173121 \\
\hline & & & & \\
\hline
\end{tabular}

(a)

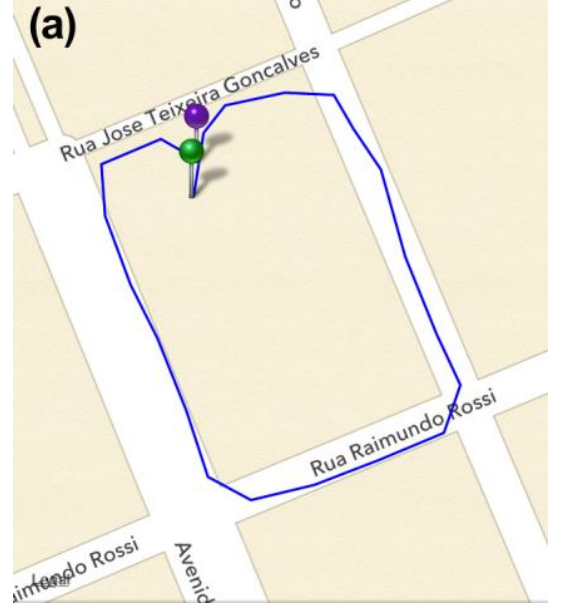

(b)

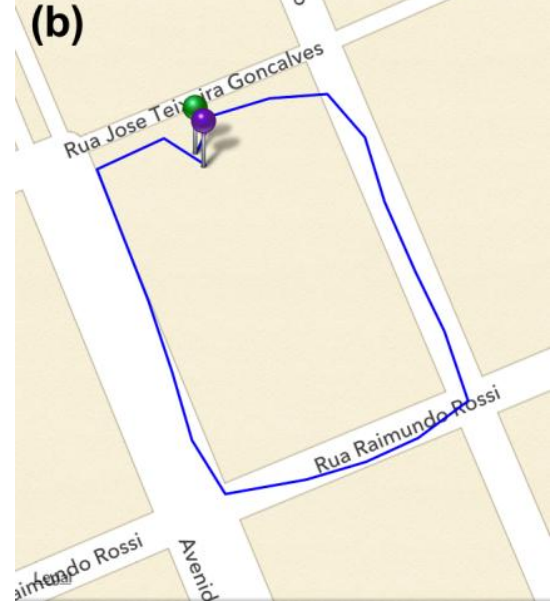

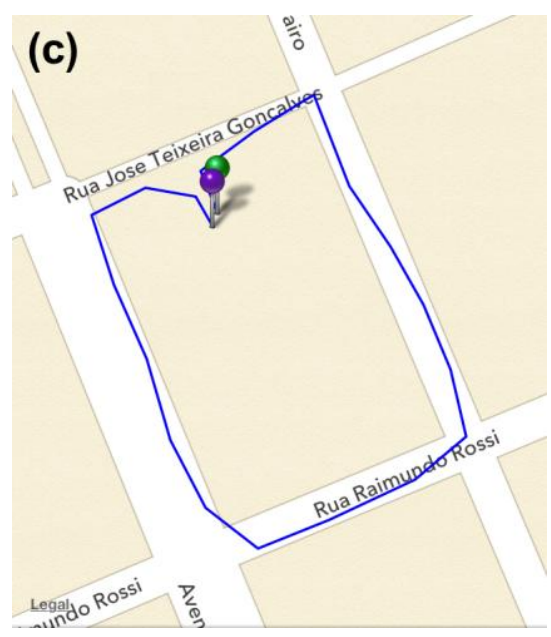

Figura 10. (a) Desenho do trajeto referente às informações do Percurso A da Tabela 2; (b) desenho do trajeto referente às informações do Percurso B da Tabela 2; (c) desenho do trajeto referente às informações do Percurso C da Tabela 2. 
A partir da comparação dos dados armazenados pela aplicação referentes a frequência cardíaca, é possível observar a existência de uma quantidade diferente de valores. Essa diferença ocorre devido ao tempo de execução da atividade física praticada, pois a pessoa praticante pode diminuir ou aumentar o seu rítimo, fazendo com que uma atividade física praticada várias vezes sobre o mesmo percurso, tenha diferentes tempos de duração. A Figura 11 ilustra a frequência cardíaca capturada durante os percursos A, B e C da atividade física praticada referente a Figura 10.

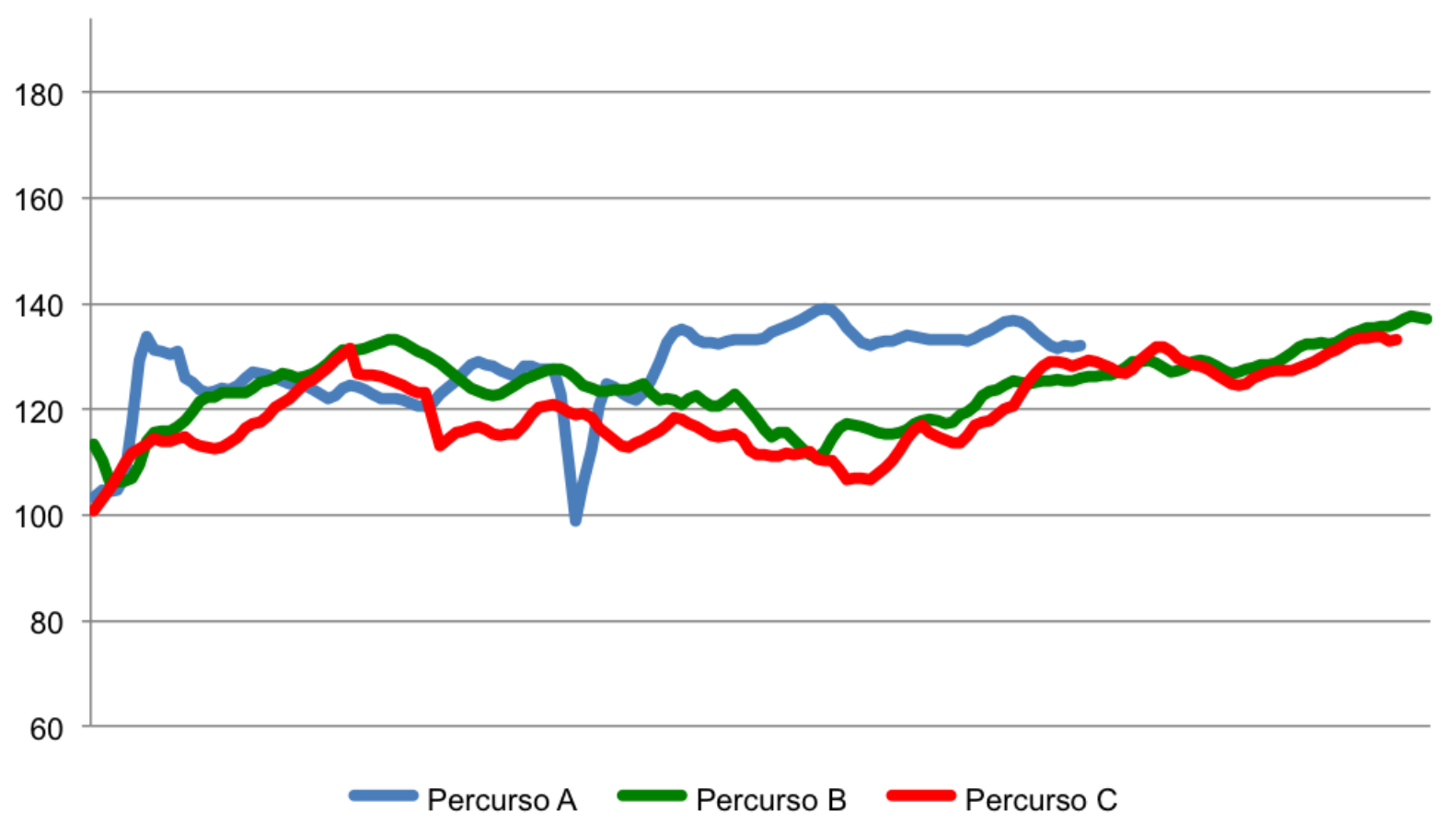

Figura 11. Comparação da frequência cardíaca capturada nos percursos $A, B$ e $C$ utilizados durante a prática de uma atividade física.

\section{CONCLUSÃO}

Com esse trabalho foi possível demonstrar como a integração de um conjunto de recursos tecnológicos pode ser utilizada para auxiliar as pessoas de terceira idade, na prática de atividades físicas, provendo um mecanismo de monitoramento à essas pessoas, visando evitar que os seus limites fisiológicos sejam excedidos, e consequentemente evitando riscos à saúde.

É importante ressaltar que a aplicação desenvolvida é apenas uma ferramenta que deve ser utilizada como auxílio à prática de atividades físicas, não substituindo o papel importante exercido pelos profissionais dessa área. 
Para trabalhos futuros, a estratégia adotada pelo uso do acelerômetro para detecção da interrupção da atividade física, pode ser unida com o deslocamento da pessoa praticante obtido a partir do GPS, tornando o recurso ainda mais preciso e confiável.

\section{REFERÊNCIAS}

AINSWORTH, B. E. et al. Compendium of physical activities: an update of activity codes and MET intensities. Medicine \& Science in Sports \& Exercise, v. 32, n. 9, Suppl., p. S498-S516, 2000.

ALMEIDA, M. B. Uma introdução ao XML, sua utilização na Internet e alguns conceitos complementares. Ciência da Informação, Brasília, v. 31, n. 2, p. 5-13, maio/ago. 2002.

ALVES, R. V. et al. Aptidão física relacionada à saúde de idosos: influência da hidroginástica. Revista Brasileira de Medicina do Esporte, 2004. Disponível em: <http://www.scielo.br/pdf/rbme/v10n1/03.p df>. Acesso em: fev. 2012.

APPLE, INC. iOS Technology overview. Disponível em: http://developer.apple.com/library/ios/\#doc umentation/Miscellaneous/Conceptual/iPho neOSTechOverview/Introduction/Introductio n.html\#//apple_ref/doc/uid/TP40007898-

CH1-SW1. Acesso em: set. 2012.

ASSIS, P. U. M. Sistema de rastreamento de veículos para empresas de transporte utilizando navegação por satélite. 2010. 121p. Monografia (Graduação) - Centro Universitário de Brasília - UniCEUB, Brasília, 2010.

CUNHA, I. C. et al. Fatores associados à prática de atividade física na população adulta de Goiânia: monitoramento por meio de entrevistas telefônicas. Revista Brasileira de Epidemiologia, v. 11, n. 3, São Paulo, p. 495-504, 2008.

DIAS, R. M. R.; GURJÃO, A. L. D.; MARUCCI, M. F. N. Benefícios do treinamento com pesos para aptidão física de idosos. Acta Fisiátrica, 2006. Disponível em: http://actafisiatrica.org.br/v1\%5Ccontrole/se cure/Arquivos/AnexosArtigos/92CC227532D 17E56E07902B254DFAD10/editoracao_vl_13 _n_02_90-95.pdf. Acesso em: fev. 2012.

FEDERIGHI, A. J. Exercício físico no idoso. Âmbito Medicina Desportiva, São Paulo, v. 03, p. 41-42, 1995.

GOYAZ, M. Vida ativa na melhor idade. Revista da Universidade Federal de Goiás UFG, vol. 5, n. 2, dez. 2003.

MARCO, M. A. Do modelo biomédico ao modelo biopsicossocial: um projeto de educação permanente. Revista Brasileira de Educação Médica, Rio de Janeiro, v.30, n.1, 2006.

MARQUES, S. A. Análise dos métodos de previsão teórica da frequência cardíaca máxima. 2009. 45p. Monografia (Graduação). Faculdade de Ciências do Desporto e Educação Física da Universidade de Coimbra, Coimbra - Portugal.

MATSUDO, S. M. Envelhecimento, atividade física e saúde. BIS, Boletim do Instituto de Saúde, São Paulo, n. 47, p. 195-209, 2009.

MILANI, A. Programando para iPhone e iPad: Aprenda a construir aplicativos para o iOS. São Paulo: Novatec, 2012. 440 p.

MCARDLE, W. D.; KATCH, F. I.; KATCH, V. L. Fisiologia do exercício: nutrição, energia e desempenho humano. 7. ed. Rio de Janeiro: Guanabara Koogan, 2011. 
NUNES, M. A. et al. Influência da percepção do peso e do índice de massa corporal nos comportamentos alimentares anormais. Revista Brasileira de Psiquiatria, São Paulo, v. 23, n. 1, mar. 2001. Disponível em: http://www.scielo.br/pdf/rbp/v23n1/a06v23 n1.pdf. Acesso em: abr. 2012.

OLIVEIRA, I. S. Física moderna para iniciados, interessados e aficionados. São Paulo: Livraria da Física, 2005. v.1. 184p.

POWERS, S. K.; HOWLEY, E. T. Fisiologia do exercício: teoria e aplicação ao condicionamento e ao desempenho. 3. ed. Manole, 2000. 527 p.

PRIESS, W. Mecanismos de escalonamento com qualidade de serviço em redes com tecnologia bluetooth. Dissertação (Mestrado em Informática) - Universidade Federal Do Rio De Janeiro - UFRJ, 2003.

RFC 791 - Internet Protocol. Disponível em: $<$ http://www.ietf.org/rfc/rfc791.txt>. Acesso em: dez. 2012.

RFC 1738 - Uniform Resource Locators (URL). Disponível em: <http://www.ietf.org/rfc/rfc1738.txt>. Acesso em: nov. 2012.

RFC 2616 - Hypertext Transfer Protocol. Disponível em: <http://www.ietf.org/rfc/rfc2616.txt>. Acesso em: nov. 2012.

ZEINDIN, D. C. A tecnologia do futuro Wi-Fi (Wireless Fidelity). Universidade Regional de Blumenau - FURB, 2003. Disponível em: http://www.inf.furb.br/ zamba/artigos/Artig o_Wireless_Uniplac_2003_V1.pdf. Acesso em: nov. 2012. 